PROCEEDINGS OF THE

AMERICAN MATHEMATICAL SOCIETY

Volume 131, Number 10, Pages 3083-3088

S 0002-9939(03)06862-X

Article electronically published on January 28, 2003

\title{
A NOTE ON THE SPECTRUM OF AN UPPER TRIANGULAR OPERATOR MATRIX
}

\author{
MOHAMED BARRAA AND MOHAMED BOUMAZGOUR
}

(Communicated by Joseph A. Ball)

\begin{abstract}
Let $M_{C}=\left[\begin{array}{cc}A & C \\ 0 & B\end{array}\right]$ be a $2 \times 2$ upper triangular operator matrix acting on the Banach space $E \oplus F$. We investigate the set of the operators $C$ for which $\sigma\left(M_{C}\right)=\sigma(A) \cup \sigma(B)$, where $\sigma($.$) denotes the spectrum.$
\end{abstract}

Let $E, F$ be complex Banach spaces and let $\mathcal{L}(F, E)$ be the space of all bounded linear operators from $F$ to $E$. We abbreviate $\mathcal{L}(E, E)$ by $\mathcal{L}(E)$. When $A \in \mathcal{L}(E)$, $B \in \mathcal{L}(F)$ and $C \in \mathcal{L}(F, E)$ are given, we denote by $M_{C}$ an operator on $E \oplus F$ of the form $\left[\begin{array}{cc}A & C \\ 0 & B\end{array}\right]$. Also, we let $L_{A}$ and $R_{B}$ denote the left (respectively right) multiplication by $A$ (respectively $B$ ). The generalized derivation induced by the operators $A$ and $B$ is defined by $\delta_{A, B}=L_{A}-R_{B}$. When $E=F$ and $A=B$, we simply write $\delta_{A, A}=\delta_{A}$.

For $T \in \mathcal{L}(E)$, we let $\sigma_{l}(T), \sigma_{r}(T), \sigma_{l e}(T), \sigma_{r e}(T), r(T), N(T)$ and $R(T)$ denote the left spectrum, right spectrum, left essential spectrum, right essential spectrum, spectral radius, null space and range of $T$ respectively. If $G$ is a subset of $\mathcal{L}(F, E)$, we denote $\operatorname{cl}[G]$ as its closure.

For bounded linear operators $A, B$ and $C$, the equality

$$
\sigma\left(M_{C}\right)=\sigma(A) \cup \sigma(B)
$$

was studied by numerous authors. In [4], it was shown that if $\sigma(A) \cap \sigma(B)$ has no interior points, then (1) is satisfied for every $C \in \mathcal{L}(F, E)$. In the Hilbert space setting, equality (1) was considered in [1, 4], where it was shown that if in particular $A$ and $B$ are normal operators, then (1) holds for every $C \in \mathcal{L}(F, E)$.

The aim of this note is the investigation of the set of operators $C \in \mathcal{L}(F, E)$ for which (11) holds.

Our main result is the following

Theorem 1. If $A \in \mathcal{L}(E)$ and $B \in \mathcal{L}(F)$, then

$$
\begin{gathered}
\sigma\left(M_{C}\right)=\sigma(A) \cup \sigma(B) \\
\text { for every } C \in \operatorname{cl}\left[R\left(\delta_{A, B}\right)+N\left(\delta_{A, B}\right)+\bigcup_{\lambda \in \mathbb{C}} N\left(L_{A-\lambda}\right)+\bigcup_{\lambda \in \mathbb{C}} N\left(R_{B-\lambda}\right)\right] .
\end{gathered}
$$

Received by the editors March 1, 2002 and, in revised form, April 23, 2002.

1991 Mathematics Subject Classification. Primary 47A10, 47A55, 47B47.

Key words and phrases. Spectrum, $2 \times 2$ upper triangular operator matrix, generalized derivation. 
As a corollary of Theorem[1, we have

Corollary 2. Assume that $E$ and $F$ are Hilbert spaces. If $A \in \mathcal{L}(E)$ and $B \in \mathcal{L}(F)$ satisfy the following conditions:

i) $\sigma_{r e}(A) \cap \sigma_{l e}(B)=\emptyset$,

ii) there exists no nonzero trace class operator $X$ such that $B X=X A$, then

$$
\sigma\left(M_{C}\right)=\sigma(A) \cup \sigma(B) \quad \text { for every } C \in \mathcal{L}(F, E) .
$$

Proof. Since $R\left(\delta_{A, B}\right)$ is norm dense (see [2]), the result follows from Theorem 1

For the proof of Theorem 1, we need some auxiliary results. We begin with the following well known lemma.

Lemma 3. If $A \in \mathcal{L}(E), B \in \mathcal{L}(F)$ and $C \in \mathcal{L}(F, E)$, then

$$
\sigma_{l}(A) \cup \sigma_{r}(B) \subseteq \sigma\left(M_{C}\right) \subseteq \sigma(A) \cup \sigma(B) .
$$

Proof. For every $\lambda \in \mathbb{C}$, we have

$$
M_{C}-\lambda=\left[\begin{array}{cc}
I & 0 \\
0 & B-\lambda
\end{array}\right]\left[\begin{array}{cc}
I & C \\
0 & I
\end{array}\right]\left[\begin{array}{cc}
A-\lambda & 0 \\
0 & I
\end{array}\right] .
$$

Since $\left[\begin{array}{ll}I & C \\ 0 & I\end{array}\right]$ is invertible, we derive that

$$
\lambda \notin \sigma(A) \cup \sigma(B) \Longrightarrow \lambda \notin \sigma\left(M_{C}\right) \Longrightarrow \lambda \notin \sigma_{l}(A) \cup \sigma_{r}(B) .
$$

This completes the proof.

The inclusions in (2) are proper in general. To see this, consider the following example from [1].

Example 4. Let $H$ be a Hilbert space with an orthonormal basis $\left\{e_{n}\right\}_{n \geq 1}$. Define the operators $S$ and $C$ by

$$
S e_{i}=e_{i+1}, \quad i=1,2, \cdots \text { and } C=e_{1} \otimes e_{1}=\left\langle., e_{1}\right\rangle e_{1} .
$$

If $S^{*}$ is the adjoint of $S$, then $\left[\begin{array}{cc}S & C \\ 0 & S^{*}\end{array}\right]$ is a unitary operator. Thus

$$
\sigma\left(\left[\begin{array}{cc}
S & C \\
0 & S^{*}
\end{array}\right]\right) \subseteq\{\lambda \in \mathbb{C}:|\lambda|=1\} \subsetneq \sigma(S) \cup \sigma\left(S^{*}\right)=\{\lambda \in \mathbb{C}:|\lambda| \leq 1\} .
$$

Also, we have

$$
\sigma_{l}(S) \cup \sigma_{r}\left(S^{*}\right)=\{\lambda \in \mathbb{C}:|\lambda|=1\} \subsetneq \sigma\left(\left[\begin{array}{cc}
S & 0 \\
0 & S^{*}
\end{array}\right]\right) .
$$

For operators $A \in \mathcal{L}(E)$ and $B \in \mathcal{L}(F)$, let

$$
M(A, B)=\left\{C \in \mathcal{L}(F, E): \sigma\left(M_{C}\right)=\sigma(A) \cup \sigma(B)\right\} .
$$

It is clear that $0 \in M(A, B)$ and $M(A, B)=M(A-\lambda, B-\lambda)$ for all complex number $\lambda$. By using the operators $S, S^{*}$ and $C$ in Example 4 , we see that the equality $M(A, B)=M(B, A)$ fails to be true in general.

The set $M(A, B)$ is norm closed. Indeed, let $\left\{C_{n}\right\}_{n}$ be a sequence in $M(A, B)$ such that $C_{n} \longrightarrow C$ as $n \longrightarrow \infty$ for some $C \in \mathcal{L}(F, E)$. Then $M_{C_{n}} \longrightarrow M_{C}$ as $n \longrightarrow \infty$. Thus, the upper semi-continuity of the spectrum (see [3, Problem 86]) implies that $\sigma\left(M_{C}\right)=\sigma(A) \cup \sigma(B)$, that is, $C \in M(A, B)$. 
The following result will be useful in the sequel.

Lemma 5. Let $T, N \in \mathcal{L}(E)$ such that $N^{2}=0$ and $N \neq 0$. If either $T N=0$ or $N T=0$, then

$$
\sigma(T+N)=\sigma(T)
$$

Proof. The proofs for the statements $T N=0$ and $N T=0$ are similar, therefore we may assume that $T N=0$. Observe first that since $(T+N) N=0$ and $N \neq 0$, then $0 \in \sigma(T+N) \cap \sigma(N)$. Next, let $\lambda \in \mathbb{C} \backslash\{0\}$. Since $T N=N^{2}=0$, we have that $N-\lambda$ is invertible, and

$$
-\lambda^{-1}(T-\lambda)(N-\lambda)=T+N-\lambda
$$

From this, we conclude that $\lambda \in \sigma(T+N)$ if and only if $\lambda \in \sigma(T)$. Consequently,

$$
\sigma(T+N)=\sigma(T)
$$

The proof is finished.

The next lemma is well known, so its proof will be omitted.

Lemma 6. Let $T, N \in \mathcal{L}(E)$ such that $\sigma(N)=\{0\}$ and $T N=N T$. Then $\sigma(T+N)=\sigma(T)$.

Using Lemma 5 and Lemma 6 we prove

Lemma 7. Let $C \in M(A, B)$ and $D \in \bigcup_{\lambda \in \mathbb{C}} N\left(L_{A-\lambda}\right) \cup \bigcup_{\lambda \in \mathbb{C}} N\left(R_{B-\lambda}\right)$. Then $C+D \in M(A, B)$.

Proof. Suppose $D \in N\left(L_{A-\lambda}\right)$ for some $\lambda$; then $(A-\lambda) D=0$. Write $M_{C+D}-\lambda=$ $M_{C}-\lambda+\left[\begin{array}{ll}0 & D \\ 0 & 0\end{array}\right]$. Since $\left[\begin{array}{ll}0 & D \\ 0 & 0\end{array}\right]^{2}=0$ and $\left(M_{C}-\lambda\right)\left[\begin{array}{ll}0 & D \\ 0 & 0\end{array}\right]=0$, we deduce from Lemma 5 that

$$
\sigma\left(M_{C+D}-\lambda\right)=\sigma\left(M_{C}-\lambda\right)=\sigma(A-\lambda) \cup \sigma(B-\lambda)
$$

that is, $C+D \in M(A, B)$.

By a similar argument, we show that if $D \in \bigcup_{\lambda \in \mathbb{C}} N\left(R_{B-\lambda}\right)$, then $C+D \in$ $M(A, B)$.

The proof is complete.

Lemma 8. If $A \in \mathcal{L}(E)$ and $B \in \mathcal{L}(F)$, then

$$
R\left(\delta_{A, B}\right)+N\left(\delta_{A, B}\right) \subseteq M(A, B) .
$$

Proof. Let $C \in R\left(\delta_{A, B}\right)$ and $D \in N\left(\delta_{A, B}\right)$. A straightforward computation shows that $M_{C}\left[\begin{array}{ll}0 & D \\ 0 & 0\end{array}\right]=\left[\begin{array}{ll}0 & D \\ 0 & 0\end{array}\right] M_{C}$. Since $\left[\begin{array}{ll}0 & D \\ 0 & 0\end{array}\right]^{2}=0$, Lemma 6 implies that

$$
\sigma\left(M_{C+D}\right)=\sigma\left(M_{C}\right)
$$

On the other hand, since $C \in R\left(\delta_{A, B}\right)$, there is an operator $X \in \mathcal{L}(E)$ such that $C=A X-X B$. Therefore, $M_{C}$ is similar to $\left[\begin{array}{cc}A & 0 \\ 0 & B\end{array}\right]$, where the similarity is implemented by the invertible operator $\left[\begin{array}{cc}I & X \\ 0 & I\end{array}\right]$. Consequently, we have

$$
\sigma\left(M_{C}\right)=\sigma(A) \cup \sigma(B) .
$$

Combining (41) and (5) shows that $C+D \in M(A, B)$, which completes the proof. 
Proof of Theorem 1. Let $C=C_{1}+C_{2}+C_{3}+C_{4}$, where $C_{1} \in R\left(\delta_{A, B}\right), C_{2} \in$ $N\left(\delta_{A, B}\right), C_{3} \in \bigcup_{\lambda \in \mathbb{C}} N\left(L_{A-\lambda}\right)$ and $C_{4} \in \bigcup_{\lambda \in \mathbb{C}} N\left(R_{B-\lambda}\right)$. By Lemma $8, C_{1}+C_{2} \in$ $M(A, B)$. Now Lemma 7 can be used twice. It shows that

$$
\sigma\left(M_{C}\right)=\sigma\left(M_{C_{1}+C_{2}+C_{3}}\right)=\sigma(A) \cup \sigma(B) .
$$

Therefore the result follows by the closedness of $M(A, B)$. This ends the proof.

Remark 1. If given $A, B$ and $C$ such that (1) holds, then using the same reasoning as in the above we check that $C+D \in M(A, B)$ for every

$$
D \in \operatorname{cl}\left[R\left(\delta_{A, B}\right)+N\left(\delta_{A, B}\right)+\bigcup_{\lambda \in \mathbb{C}} N\left(L_{A-\lambda}\right) \cup \bigcup_{\lambda \in \mathbb{C}} N\left(R_{B-\lambda}\right)\right] .
$$

As an application of Theorem 1 we have the next example.

Example 9. Let $A_{1} \in \mathcal{L}(E)$ and $B_{1} \in \mathcal{L}(F)$. If $A \in \mathcal{L}(E \oplus E)$ and $B \in \mathcal{L}(F \oplus F)$ are the operators defined by $A=\left[\begin{array}{cc}A_{1} & 0 \\ 0 & 0\end{array}\right]$ and $B=\left[\begin{array}{cc}0 & 0 \\ 0 & B_{1}\end{array}\right]$, then $\sigma\left(M_{C}\right)=\sigma(A) \cup \sigma(B)$ for every $C \in \mathcal{L}(F \oplus F, E \oplus E)$ of the form $\left[\begin{array}{ll}C_{1} & C_{2} \\ C_{3} & C_{4}\end{array}\right]$ such that

$$
C_{2} \in \operatorname{cl}\left[R\left(\delta_{A_{1}, B_{1}}\right)+N\left(\delta_{A_{1}, B_{1}}\right)\right] .
$$

Indeed, write

$$
C=\left[\begin{array}{cc}
C_{1} & 0 \\
0 & 0
\end{array}\right]+\left[\begin{array}{cc}
0 & C_{2} \\
0 & 0
\end{array}\right]+\left[\begin{array}{cc}
0 & 0 \\
C_{3} & C_{4}
\end{array}\right]
$$

Since

we have

$$
\left[\begin{array}{cc}
C_{1} & 0 \\
0 & 0
\end{array}\right]\left[\begin{array}{cc}
0 & 0 \\
0 & B_{1}
\end{array}\right]=\left[\begin{array}{ll}
0 & 0 \\
0 & 0
\end{array}\right],
$$

$$
\left[\begin{array}{cc}
C_{1} & 0 \\
0 & 0
\end{array}\right] \in N\left(R_{B}\right) .
$$

Also, since $C_{2} \in \operatorname{cl}\left[R\left(\delta_{A_{1}, B_{1}}\right)+N\left(\delta_{A_{1}, B_{1}}\right)\right]$, it follows that

$$
\left[\begin{array}{cc}
0 & C_{2} \\
0 & 0
\end{array}\right] \in \operatorname{cl}\left[R\left(\delta_{A, B}\right)+N\left(\delta_{A, B}\right)\right] .
$$

Next, we have

$$
\left[\begin{array}{cc}
A_{1} & 0 \\
0 & 0
\end{array}\right]\left[\begin{array}{cc}
0 & 0 \\
C_{3} & C_{4}
\end{array}\right]=\left[\begin{array}{ll}
0 & 0 \\
0 & 0
\end{array}\right] .
$$

Thus

$$
\left[\begin{array}{cc}
0 & 0 \\
C_{3} & C_{4}
\end{array}\right] \in N\left(L_{A}\right) .
$$

Consequently, Theorem 1 implies that $C \in M(A, B)$.

If, in particular, $A_{1}=S$ and $B_{1}=S^{*}$, where $S$ is the operator defined in Example 4, we see that $\sigma_{r}(A) \cap \sigma_{l}(B)$ may be large in a certain sense.

We conclude with the next proposition.

Proposition 10. Let $A \in \mathcal{L}(E), B \in \mathcal{L}(F)$ and $C \in \mathcal{L}(F, E)$. Assume that there exists some $\lambda \in(\sigma(A) \cup \sigma(B)) \backslash \sigma\left(M_{C}\right)$. Then $\sigma_{r}(A) \cap \sigma_{l}(B) \backslash\left(\sigma_{l}(A) \cup \sigma_{r}(B)\right)$ contains a neighborhood of $\lambda$. 
Proof. Without loss of generality we may assume that $\lambda=0$. Since $0 \notin \sigma\left(M_{C}\right)$, Lemma 3 implies that $0 \notin \sigma_{l}(A)$. Hence there is an operator $U \in \mathcal{L}(E)$ such that $U A=I$ ( $I$ : the identity operator). Let $\epsilon_{1}=\frac{1}{r(U)}$ and let $|\lambda|<\epsilon_{1}$. Then $I-\lambda U$ is invertible, and

$$
U(I-\lambda U)^{-1}(A-\lambda)=I .
$$

On the other hand we deduce from (3) that $A U \neq I$. Thus

$$
(A-\lambda) U(I-\lambda U)^{-1}=(A U-\lambda U)(I-\lambda U)^{-1} \neq I .
$$

From (6) and (7) we derive that

$$
\lambda \in \sigma_{r}(A) \backslash \sigma_{l}(A) .
$$

Consequently, we have

$$
B\left(0, \epsilon_{1}\right) \subseteq \sigma_{r}(A) \backslash \sigma_{l}(A) .
$$

By a similar argument, we claim that there exists $\epsilon_{2}>0$ such that

$$
B\left(0, \epsilon_{2}\right) \subseteq \sigma_{l}(B) \backslash \sigma_{r}(B) .
$$

Let $\epsilon=\min \left(\epsilon_{1}, \epsilon_{2}\right)$. Then

$$
B(0, \epsilon) \subseteq \sigma_{r}(A) \cap \sigma_{l}(B) \backslash\left(\sigma_{l}(A) \cup \sigma_{r}(B)\right) .
$$

This ends the proof.

Corollary 11. If $\sigma_{r}(A) \cap \sigma_{l}(B) \backslash\left(\sigma_{l}(A) \cup \sigma_{r}(B)\right)$ has no interior points, then

$$
M(A, B)=\mathcal{L}(F, E) .
$$

If in particular $E=F$ and $A=B$, then

$$
M(A, A)=\mathcal{L}(E) .
$$

Remark 2. Suppose $E$ and $F$ are Hilbert spaces. It is clear that, for given operators $A$ and $B, \sigma_{r}(A) \cap \sigma_{l}(B) \backslash\left(\sigma_{l}(A) \cup \sigma_{r}(B)\right) \subseteq \sigma_{p}\left(A^{*}\right)^{*} \cap \sigma_{p}(B)$, where $\sigma_{p}\left(A^{*}\right)^{*}$ denotes the set of the complex conjugates of points of $\sigma_{p}\left(A^{*}\right)$. We conclude from Proposition 10 that if $\sigma_{p}\left(A^{*}\right)^{*} \cap \sigma_{p}(B)$ has no interior points, then $M(A, B)=$ $\mathcal{L}(F, E)$.

Let us give an example showing that the converse of Theorem 1 is not true.

Example 12. Let $A \in \mathcal{L}(E)$ such that $A^{2}=0$ and $A \neq 0$. A simple computation shows that $\delta_{A}^{3}=0$. Thus $R\left(\delta_{A}\right) \subseteq N\left(\delta_{A}^{2}\right)$. If $\lambda \in \mathbb{C}$ and $X \in N\left(L_{A-\lambda}\right)$, then $A X=\lambda X$. Since $A^{2}=0$, we conclude that $A X=0$. Hence $X \in N\left(\delta_{A}^{2}\right)$. Therefore

$$
\bigcup_{\lambda \in \mathbb{C}} N\left(L_{A-\lambda}\right) \subseteq N\left(\delta_{A}^{2}\right)
$$

A similar reasoning shows that

$$
\bigcup_{\lambda \in \mathbb{C}} N\left(R_{A-\lambda}\right) \subseteq N\left(\delta_{A}^{2}\right)
$$

Consequently, we have

$$
\begin{aligned}
& c l\left[R\left(\delta_{A}\right)+N\left(\delta_{A}\right)+\bigcup_{\lambda \in \mathbb{C}} N\left(L_{A-\lambda}\right)+\bigcup_{\lambda \in \mathbb{C}} N\left(R_{A-\lambda}\right)\right] \\
& \subseteq N\left(\delta_{A}^{2}\right) \subsetneq M(A, A)=\mathcal{L}(E) .
\end{aligned}
$$




\section{ACKNOWLEDGMENT}

The authors are grateful to the referee for valuable comments and suggestions.

\section{REFERENCES}

1. H.K. Du and J. Pan, Perturbation of spectrums of $2 \times 2$ operator matrices, Proc. Amer. Math. Soc. 121(1994), 761-776. MR 94i:47004

2. L. Fialkow, $A$ note on the range of the operator $X \longrightarrow A X-X B$, Illinois J. Math. 25(1981), 112-124. MR 84b:47021

3. P.R. Halmos, A Hilbert space problem book, Springer Verlag, New York, 1973. MR 84e:47001

4. J.K. Han, H.Y. Lee and W.Y. Lee, Invertible completions of $2 \times 2$ upper triangular operator matrices, Proc. Amer. Math. Soc. 129(2000), 119-123. MR 2000c:47003

Département de Mathématiques, Faculté des Sciences Semlalia, B.P 2390, Marrakech, MAROC

E-mail address: barraa@hotmail.com

Département de Mathématiques, Faculté des Sciences Semlalia, B.P 2390, Marrakech, MAROC

E-mail address: boumazgour@ucam.ac.ma

Current address: Département de Mathématiques et Statistique, Pavillon Alexandre Vachon, Université Laval, Québec, Canada G1K 7P4

E-mail address: boumazgo@mat.ulaval.ca 